\title{
An Algorithm for the Preclinical Screening of Anticancer Drugs Effective against Brain Tumors
}

\author{
Juan Sebastian Yakisich \\ Department of Clinical Neuroscience, Karolinska University Hospital, Karolinska Institute, SE-141 86 Stockholm, Sweden \\ Correspondence should be addressed to Juan Sebastian Yakisich, sebastian.yakisich@ki.se
}

Received 23 April 2012; Accepted 11 June 2012

Academic Editors: W. Jäger, T. Kumai, and T. B. Vree

Copyright ( $) 2012$ Juan Sebastian Yakisich. This is an open access article distributed under the Creative Commons Attribution License, which permits unrestricted use, distribution, and reproduction in any medium, provided the original work is properly cited.

\begin{abstract}
The anticancer drugs screening program is a long and expensive process. It is estimated that only $5 \%$ of drugs entering clinical trials are approved by the FDA. Moreover, many of the drugs that enter clinical trials are often of limited use in clinical practice, and most cancers remain untreatable. Brain tumors are particularly difficult to treat due to the presence of the blood brain barrier that limits the penetration of anticancer drugs. Additionally the isolation from most brain tumors of putative cancer stem cells and novel models of cancer stem cell biology suggest that anticancer drugs should be delivered for prolonged time and at higher concentrations to deplete any potential tumorigenic cell. In this paper, current concepts of cancer stem cell biology and novel concepts of anticancer drugs screening are integrated to develop a seven-steps algorithm as a guideline for the preclinical evaluation of active compounds for the treatment of brain tumors. The flexibility of the algorithm allows the inclusion of alternative studies to exhaustively investigate anticancer drugs and creates multiple opportunities where decisions to engage or not in early clinical trials can be made providing a useful tool for translational research in neurooncology.
\end{abstract}

\section{Introduction}

The treatment of primary and metastatic human brain tumors (HBTs) faces important challenges due to the presence of the blood brain barrier that limits the diffusion of anticancer drugs [1]. For instance, while the serum concentrations of etoposide after a dose of $50 \mathrm{mg} / \mathrm{m}^{2}$ can reach values $>1 \mathrm{mg} / \mathrm{L}(\sim 1.7 \mu \mathrm{M})$ [2], the average cerebro spinal fluid (CSF) concentrations after a dose $300 \mathrm{mg} / \mathrm{m}^{2}$ i.v. was $0.175 \mu \mathrm{M}$; (range, 0.066 to 2.12) [3]. As expected, etoposide alone has been of limited use for brain tumors. The isolation of cancer stem cells (CSCs) from a variety of primary brain tumors including gliomas [4], oligodendrogliomas, meningiomas $[5,6]$, and other tumors that frequently metastasize into the brain further complicates this scenario. In particular, novel models of cancer biology support the idea that CSCs and non-CSCs can interconvert into each other [7-11]. Thus, to cure cancer all malignant cells should be eliminated at once otherwise, any surviving cell that is potentially tumorigenic will eventually regenerate the tumor, sometimes after long disease-free intervals. From the clinical aspect, brain tumor treatment will require the use of drugs or drugs combinations that should reach the brain tumoral tissue for extended periods of time at effective concentrations to eliminate all cancer cells. With this in mind, our lab developed several novel concepts that include (i) the regrowth concentration zero $\left(\mathrm{RC}_{0}\right)$ : defined as the minimum drug concentration that kills 100\% of cancer cells preventing regrowth when cells are re-incubated in drug free media [12], (ii) the term pankiller: defined as drugs or drugs combination that deplete cancer cells $[13,14]$, (iii) a novel two phases treatment (2PT) that in vitro was shown to be effective in eliminating all cancer cells. In this treatment regime, cells are exposed to drugs for few weeks (first phase) that eliminates most of the cancer cells leaving few surviving cells that are eliminated by treatment with a second drug (second phase). It has the advantage that the concentration required for each phase is lower than the concentration required for individual drugs to deplete all cells. For instance, while $1-5 \mu \mathrm{M}$ salinomycin alone for one week was unable to eliminate $100 \%$ of previously untreated gliomas cells, cells that survived 3-4 weeks continuous exposure to $10 \mathrm{mM}$ 
hydroxyurea were eliminated with only $0.5 \mu \mathrm{M}$ salinomycin [15].

On the other hand, there is no anticancer drug program that really fits the needs for the screening of active compounds for HBTs and in practice, clinical trials are often initiated with limited information that usually lead to a failure. In average, the cost of each failure for oncology drugs is estimated in the range of hundreds millions of dollars per drug [16]. It is then imperative to develop strategies to minimize the rate of such failures and identify drugs with higher probability to succeed in clinical trials. In this article, based in the above-mentioned current models of cancer stem cell biology and novel concepts and strategies developed in our laboratory, an integrated algorithm is proposed as a general guideline for a more rational screening of anticancer drugs for HBTs.

\section{Drug Screening Algorithm for Brain Tumors}

The proposed algorithm and its rationale for the screening of new anticancer drugs for HBTs can be summarized as follow (See Figure 1). The course to follow can be guided by a simple "Yes" or "No" response based in the success or failure, respectively, of the previous step.

Step 1. Initial Characterization of the $I C_{50}$ for Specific Cancer. In this step a variety of standard primary or commercial cancer cell lines or tridimensional systems can be used. The $\mathrm{IC}_{50}$ is a general endpoint to test the effect of drugs and gives valuable information such as potency and potential mechanism(s) of action. The $\mathrm{IC}_{50}$ can be determined by a variety of high-throughput screening methods within 34 days and allows the selection of potential active drugs from large compound libraries. A failure ("NO") at this stage means that drug investigated is ineffective as anticancer drug, and it is not worth pursuing additional steps $\left(\right.$ no-GO $\left.{ }^{1}\right)$.

Step 2. Determination of the $R C_{0}$ for Selected Compounds. Since there is no high-throughput assay available for the determination of the $\mathrm{RC}_{0}$ (that takes around 4 weeks), this step is rate limiting but may select compounds with the potential to eradicate all cancer cells at once. A failure ("NO") at this stage means that while the drug may have shown some anticancer effect during Step 1, it will not deplete all malignant cells as a single agent. Therefore, it should be withdrawn from the screening process (no-GO ${ }^{2}$ ). During Steps 1 and 2, the pharmacokinetic of the drugs should be studied in parallel to measure the concentration that actually reaches the brain tissue. If the $\mathrm{RC}_{0}$ concentration (and necessary exposure time) can be achieved in brain tissue by systemic delivery ("Yes"), one should proceed with Step 3, otherwise proceed to Step 4 (option 1) or Step 5 (option 2, optional at this stage).

Step 3. Effectiveness of ADs on Animal Models Administered by Systemic Delivery on a 1 Phase Treatment (1PT) Regime. A success at this step will lead the process to the first $\left(\mathrm{GO}^{1}\right)$ decision and a failure to Step 5 .
Step 4. Effectiveness of ADs on Animal Models Administered by Interstitial Delivery on a 1 Phase Treatment (1PT) Regime. Similar to Step 3 this study will determine the effectiveness in brain tumors and at the same time evaluate its adverse effects. Several ways to deliver ADs locally (biodegradable polymers, miniosmotic pump, and nanoparticles) can be evaluated at this stage. The local delivery of anticancer drugs by biodegradable polymers has been used in animal models [17] and in patients [18], but its uses not widespread. Miniosmotic pumps [19] and nanoparticles [20] are promising strategies for local delivery, but at present their use is limited to experimental models and they have not been translated into clinical use. A success ("Yes") at this step will lead the screening process to the second $\left(\mathrm{GO}^{2}\right)$ decision and a failure ("NO") to Step 5.

Step 5. In Vitro Effectiveness of Drugs Administered in a Two Phase Treatment (2PT) Regime. The aim of this type of screening is to find a drug combination that can be used sequentially (even at lower concentrations) to eradicate all tumor cells in vitro. While the failure of this step will lead to the third decision to stop the evaluation of that particular compound $\left(\right.$ no- $\left.-\mathrm{GO}^{3}\right)$, the success will open the possibility to pursue systemic delivery in animal models (Step 6) or interstitial in animal models (Step 7) depending on whether the intracranial concentrations of both drugs can be achieved by systemic delivery.

Step 6. Effectiveness of ADs on Animal Models Administered by Systemic Delivery on a 2 Phase Treatment (2PT) Regime. If successful it will lead to another opportunity to engage in clinical trials $\left(\mathrm{GO}^{3}\right)$. Its failure will lead to a fourth decision to stop the screening $\left(\right.$ no- $\left.\mathrm{GO}^{4}\right)$.

Step 7. Effectiveness of ADs on Animal Models Administered by Interstitial l Delivery on a 2 Phase Treatment (2PT) Regime. If successful it will lead to the last opportunity to engage in clinical trials $\left(\mathrm{GO}^{4}\right)$. Its failure will lead to a fifth decision to stop the screening $\left(\right.$ no- $\left.-\mathrm{GO}^{5}\right)$.

\section{Discussion}

The term brain tumors refers to a variety of entities with unique clinicopathological characteristics [21], and it is likely that each type of brain tumor will require a specific treatment modality. There is a plethora of model systems (cell lines and animal models) for those tumors that chemotherapy may play an important role and screening of new anticancer drugs are being actively investigated. Only for gliomas, there are several well-established cell lines commonly used in neurooncology. Barth and Kaur [22], described in detail eight rat brain tumor models (and their corresponding cell lines). Mouse models of gliomas are also available [23]. Therefore, at this level, the available resources are multiple for each specific brain tumor but there is no consensus in which cell line is the best since in general cell cultures do not represent the complex heterogeneity of human brain tumors that is the main disadvantage of cancer cell lines [24]. Despite this limitation, cell lines are very useful for 


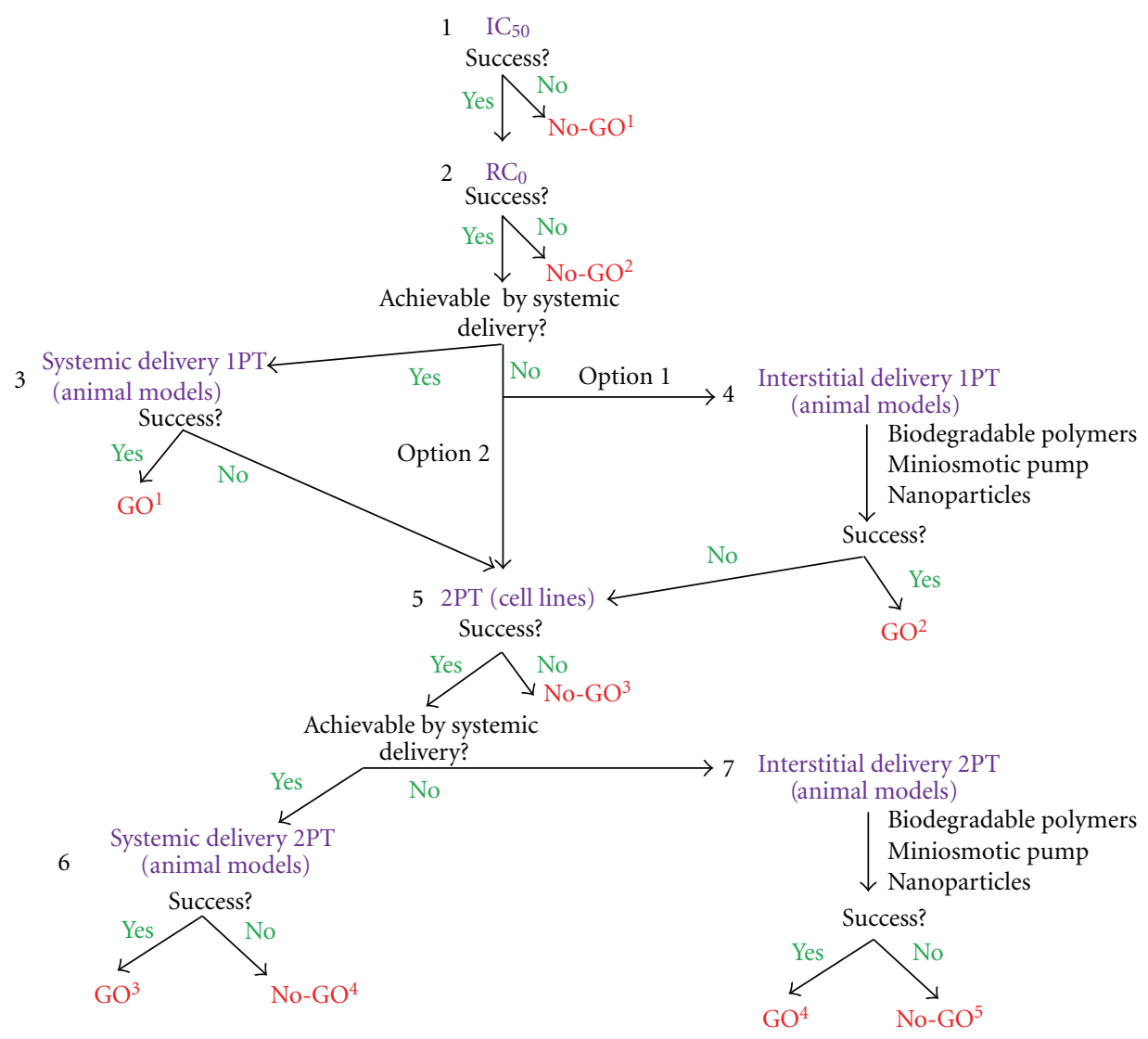

FIGURE 1: Proposed algorithm for the screening of potential anticancer drugs against human brain tumors to decide whether to engage (GO) or not (no-GO) into early (Phase I and II) clinical trials. The algorithm is composed of seven Steps 1-7 where decisions can be made. See main text for details.

the initial characterization of potential anticancer compounds for their low cost compared to other more complex systems and the feasibility to perform high-throughput screening. The selection of successful candidates ("winners") for early clinical trials is recognized as a crucial aspect of the drug screening program [25]. As seen in the algorithm depicted in Figure 1, all GO decisions are made after tests in animal models. Similar to the situation found with cell lines, there are many different animal models for each major brain tumor types (e.g., gliomas [26, 27] and meningiomas [28]) and therefore, a detailed description of these models is beyond the scope of this paper. Thus, the choice of the animal model and the endpoint parameter (e.g., overall survival or tumor size) to evaluate the response of the treatment is critical and should be chosen after careful considerations depending on the type of brain tumor under investigation. After a decision to enter a clinical trial (GO), its success is difficult to predict since there is no optimal animal model that faithfully resembles the human brain tumors $[26,28]$, and therefore there is no guarantee that a cure in animal models will be translated into a clinical success in human patients. The choice of the endpoint in the animal model should be those that monitor overall survival (or progression free survival) for long time rather than measuring the tumor size after a short period of treatment. The rationale for this statement is that a simple reduction of tumor size means that a fraction of tumoral cells is still viable, and those cells even if they become senescent have the potential to escape the senescence state and regrow [15]. The basic algorithm (Figure 1) can be complemented with additional studies that can provide valuable information and/or improve the outcome of specific steps: (i) to improve the bioavailability, the drugs can be administered concomitant with strategies aimed to disrupt $[29,30]$, or bypass the BBB by targeted therapy [31] or transnasal delivery [32], (ii) pharmacokinetics of radiolabelled compounds by positron emission tomography [33-35] can be useful to assess interspecies difference in metabolism of anticancer drugs [34]. In a similar way phase 0 clinical trials can also give valuable information [36], and they could be feasible to introduce as an additional step after any GO decision and prior to the phase I clinical trial. It is important to notice that these additional studies do not change the structure of the algorithm. They may only favor the YES or NO answer. For instance, if standard systemic delivery (e.g., intravenously) is not enough to achieve the $\mathrm{RC}_{0}$ concentration in the brain tissue, one should follow the NO direction (after Steps 2 or 5). However, if the drugs is administered by concomitant disruption of the BBB (or any of the strategies mentioned above), it may be possible to achieve the $\mathrm{RC}_{0}$ concentration and consequently follow 
the YES direction. This algorithm is intended to improve the success rate of the transition between the preclinical stage and early clinical trials (phase I and II) where toxicity and antitumor activity are the main endpoints [36]. One should be aware that many drugs fail late in development (often in Phase III trials) due to unexpected issues related to safety, efficacy, and confounded outcomes [37] that can be very difficult to predict even after successful Phase II trials. On the other hand, early and right decision to not engage into a clinical trial (No-GO) will save time and resources that can be used to give other compounds the chance to be evaluated as anticancer drugs. Right no-GO decisions are also important to prevent the wrong elimination of potential useful drugs from the ADSP. The inclusion of the $\mathrm{RC}_{0}$ and the $2 \mathrm{PT}$ in the core algorithm allows additional opportunities for a drug to be exhaustively tested before being labeled as ineffective for HBTs. Finally, future improvements on methodologies used at each one of the seven steps would improve the chances for a particular drug or drug combination to succeed in clinical trials.

\section{Conclusions}

The proposed algorithm combines in a rational way results from experimental studies of diverse nature (proliferation assays, pharmacokinetics, response in animal models of BTs) to create step-by-step guidelines for anticancer drug screening of potential active compounds against HBTs. In particular, the basic proposed algorithm is composed of 7 seven potential points (steps) where drugs or drugs combinations can be experimentally tested. The progression in the algorithm is guided by" Yes" or "No" responses to previous results that provide four opportunities where decisions can be made to enter a clinical trial $\left(\mathrm{GO}^{1}-\mathrm{GO}^{4}\right)$ or five opportunities where the screening process should stop $\left(\right.$ no- $\left.\mathrm{GO}^{1}-\mathrm{no}-\mathrm{GO}^{5}\right)$. Its flexibility allows the inclusion of additional studies that creates more opportunities to take GO or no-GO decisions to enter clinical trials and can be a useful guideline for the identification of active compounds and optimization of therapeutic regimes for HBTs.

\section{Conflict of Interests}

The author declares no conflict of interests.

\section{Acknowledgments}

Research in the author's lab is supported by grants from the Swedish Research Council and the Karolinska Institute.

\section{References}

[1] D. Fortin, "The blood-brain barrier: its influence in the treatment of brain tumors metastases," Current Cancer Drug Targets, vol. 12, no. 3, pp. 247-259, 2012.

[2] J. D. Hainsworth, "Extended-schedule oral etoposide in selected neoplasms and overview of administration and scheduling issues," Drugs, vol. 58, no. 3, pp. 51-56, 1999.
[3] M. V. Relling, H. H. Mahmoud, C. H. Pui et al., "Etoposide achieves potentially cytotoxic concentrations in CSF of children with acute lymphoblastic leukemia," Journal of Clinical Oncology, vol. 14, no. 2, pp. 399-404, 1996.

[4] T. Kondo, T. Setoguchi, and T. Taga, "Persistence of a small subpopulation of cancer stem-like cells in the C6 glioma cell line," Proceedings of the National Academy of Sciences of the United States of America, vol. 101, no. 3, pp. 781-786, 2004.

[5] D. Y. Hueng, H. K. Sytwu, S. M. Huang, C. Chang, and H. I. $\mathrm{Ma}$, "Isolation and characterization of tumor stem-like cells from human meningiomas," Journal of Neuro-Oncology, vol. 104, no. 1, pp. 45-53, 2010.

[6] P. Rath, D. C. Miller, N. S. Litofsky et al., "Isolation and characterization of a population of stem-like progenitor cells from an atypical meningioma," Experimental and Molecular Pathology, vol. 90, no. 2, pp. 179-188, 2011.

[7] M. Cruz, A. Siden, D. R. Tasat, and J. S. Yakisich, "Are all glioma cells cancer stem cells?" Journal of Cancer Science and Therapy, vol. 2, no. 4, pp. 100-106, 2010.

[8] M. A. Hatiboglu, J. Wei, A. S. G. Wu, and A. B. Heimberger, "Immune therapeutic targeting of glioma cancer stem cells," Targeted Oncology, vol. 5, no. 3, pp. 217-227, 2010.

[9] D. R. Laks, K. Visnyei, and H. I. Kornblum, "Brain tumor stem cells as therapeutic targets in models of glioma," Yonsei Medical Journal, vol. 51, no. 5, pp. 633-640, 2010.

[10] Y. Li and J. Laterra, "Cancer stem cells: distinct entities or dynamically regulated phenotypes?" Cancer Research, vol. 72, no. 3, pp. 576-580, 2012.

[11] L. Vermeulen, F. de Sousa, E. Melo, D. J. Richel, and J. P. Medema, "The developing cancer stem-cell model: clinical challenges and opportunities," The Lancet Oncology, vol. 13, no. 2, pp. 83-89, 2012.

[12] D. Avramidis, M. Cruz, Å. Sidén, D. R. Tasat, and J. S. Yakisich, "Regrowth Concentration Zero (RC0) as complementary endpoint parameter to evaluate compound candidates during preclinical drug development for cancer treatment," Journal of Cancer Science \& Therapy, vol. 1, pp. 19-24, 2009.

[13] Z. M. Delwar, D. Avramidis, E. Follin et al., "Cytotoxic effect of menadione and sodium orthovanadate in combination on human glioma cells," Investigational New Drugs. In press.

[14] M. F. Vita, N. Nagachar, D. Avramidis et al., "Pankiller effect of prolonged exposure to menadione on glioma cells: potentiation by vitamin C," Investigational New Drugs, vol. 29, no. 6, pp. 1314-1320, 2010.

[15] Z. M. Delwar, D. Avramidis, A. Siden, M. Cruz, and J. S. Yakisich, "Depletion of drug-surviving glioma cells by a second phase treatment with low concentration of salinomycin," Drugs and Therapy Studies, vol. 1, p. e7, 2011.

[16] J. A. Di Masi and H. G. Grabowski, "Economics of new oncology drug development," Journal of Clinical Oncology, vol. 25, no. 2, pp. 209-216, 2007.

[17] V. R. Recinos, B. M. Tyler, K. Bekelis et al., "Combination of intracranial temozolomide with intracranial carmustine improves survival when compared with either treatment alone in a rodent glioma model," Neurosurgery, vol. 66, no. 3, pp. 530-537, 2010.

[18] P. Miglierini, M. Bouchekoua, B. Rousseau, P. Dam Hieu, J. Malhaire, and O. Pradier, "Impact of the per-operatory application of GLIADEL wafers (BCNU, carmustine) in combination with temozolomide and radiotherapy in patients with glioblastoma multiforme: efficacy and toxicity," Clinical Neurology and Neurosurgery. In press.

[19] G. Xi, B. Mania-Farnell, V. Rajaram et al., "Efficacy of interstitial continuous vincristine infusion in a bioluminescent 
rodent intracranial tumor model," Journal of Neuro-Oncology, vol. 106, no. 2, pp. 261-270, 2012.

[20] R. N. Saha, S. Vasanthakumar, G. Bende, and M. Snehalatha, "Nanoparticulate drug delivery systems for cancer chemotherapy," Molecular Membrane Biology, vol. 27, no. 7, pp. 215-231, 2010.

[21] D. N. Louis, H. Ohgaki, O. D. Wiestler et al., “The 2007 WHO classification of tumours of the central nervous system," Acta Neuropathologica, vol. 114, no. 2, pp. 97-109, 2007.

[22] R. F. Barth and B. Kaur, "Rat brain tumor models in experimental neuro-oncology: the C6, 9L, T9, RG2, F98, BT4C, RT2 and CNS-1 gliomas," Journal of Neuro-Oncology, vol. 94, no. 3, pp. 299-312, 2009.

[23] E. Binello, Z.A. Qadeer, H.P. Kothari, L. Emdad, and I.M. Germano, "Stemness of the CT-2A Immunocompetent mouse brain tumor model: characterization in vitro," Journal of Cancer, vol. 3, pp. 166-174, 2012.

[24] H. Karlsson, M. Fryknäs, R. Larsson, and P. Nygren, "Loss of cancer drug activity in colon cancer HCT-116 cells during spheroid formation in a new 3-D spheroid cell culture system," Experimental Cell Research, vol. 318, no. 13, pp. 1577-1585, 2012.

[25] R. Goodwin, G. Giaccone, H. Calvert, M. Lobbezoo, and E. A. Eisenhauer, "Targeted agents: how to select the winners in preclinical and early clinical studies?" European Journal of Cancer, vol. 48, no. 2, pp. 170-178, 2012.

[26] V. L. Jacobs, P. A. Valdes, W. F. Hickey, and J. A. De Leo, "Current review of in vivo GBM rodent models: emphasis on the CNS-1 tumour model," ASN Neuro, vol. 3, no. 3, Article ID e00063, 2011.

[27] T. S. Jones and E. C. Holland, "Animal models for glioma drug discovery," Expert Opinion on Drug Discovery, vol. 6, no. 12, pp. 1271-1283, 2011.

[28] M. Kalamarides, M. Peyre, and M. Giovannini, "Meningioma mouse models," Journal of Neuro-Oncology, vol. 99, no. 3, pp. 325-331, 2010.

[29] S. J. Madsen and H. Hirschberg, "Site-specific opening of the blood-brain barrier," Journal of Biophotonics, vol. 3, no. 5-6, pp. 356-367, 2010.

[30] C. Y. Ting, C. H. Fan, H. L. Liu et al., "Concurrent blood-brain barrier opening and local drug delivery using drug-carrying microbubbles and focused ultrasound for brain glioma treatment," Biomaterials, vol. 33, no. 2, pp. 704-712, 2012.

[31] P. Zhang, L. Hu, Q. Yin, L. Feng, and Y. Li, "Transferrinmodified c[RGDfK]-paclitaxel loaded hybrid micelle for sequential blood-brain barrier penetration and glioma targeting therapy," Molecular Pharmacology, vol. 9, no. 6, pp. 15901598, 2012.

[32] T. Shingaki, D. Inoue, T. Furubayashi et al., "Transnasal delivery of methotrexate to brain tumors in rats: a new strategy for brain tumor chemotherapy," Molecular Pharmaceutics, vol. 7, no. 5, pp. 1561-1568, 2010.

[33] O. C. Hutchinson, D. R. Collingridge, H. Barthel, P. M. Price, and E. O. Aboagye, "Pharmacokinetics of radiolabelled anticancer drugs for positron emission tomography," Current Pharmaceutical Design, vol. 9, no. 11, pp. 917-929, 2003.

[34] S. Osman, S. K. Luthra, F. Brady et al., "Studies on the metabolism of the novel antitumor agent [N-methyl- 11C]N[2-(dimethylamino)ethyl] acridine-4-carboxamide in rats and humans prior to phase I clinical trials," Cancer Research, vol. 57, no. 11, pp. 2172-2180, 1997.

[35] A. Saleem, E. O. Aboagye, J. C. Matthews, and P. M. Price, "Plasma pharmacokinetic evaluation of cytotoxic agents radiolabelled with positron emitting radioisotopes," Cancer Chemotherapy and Pharmacology, vol. 61, no. 5, pp. 865$873,2008$.

[36] J. Arrondeau, H. K. Gan, A. R. Razak, X. Paoletti, and C. Le Tourneau, "Development of anti-cancer drugs," Discovery medicine, vol. 10, no. 53, pp. 355-362, 2010.

[37] G. J. Kelloff and C. C. Sigman, "New science-based endpoints to accelerate oncology drug development," European Journal of Cancer, vol. 41, no. 4, pp. 491-501, 2005. 

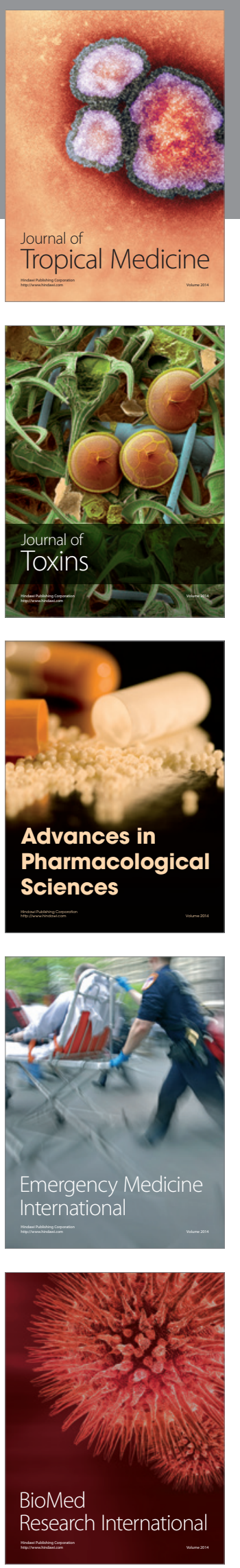
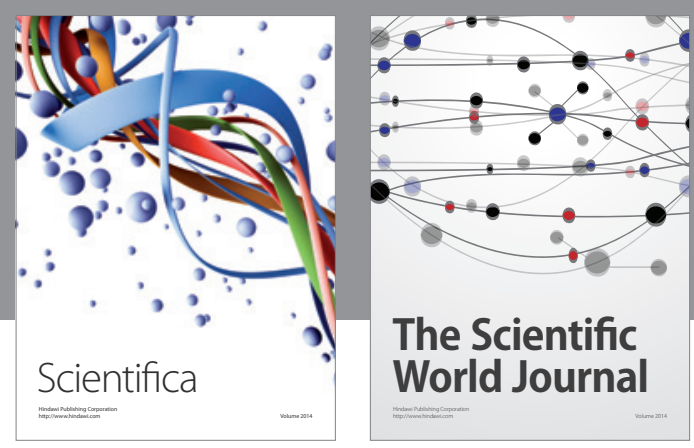

The Scientific World Journal
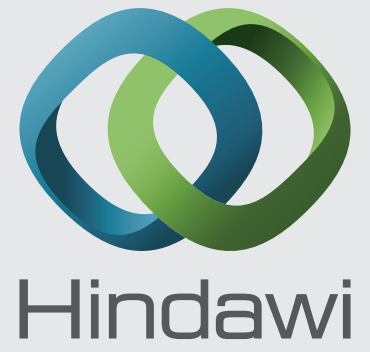

Submit your manuscripts at

http://www.hindawi.com
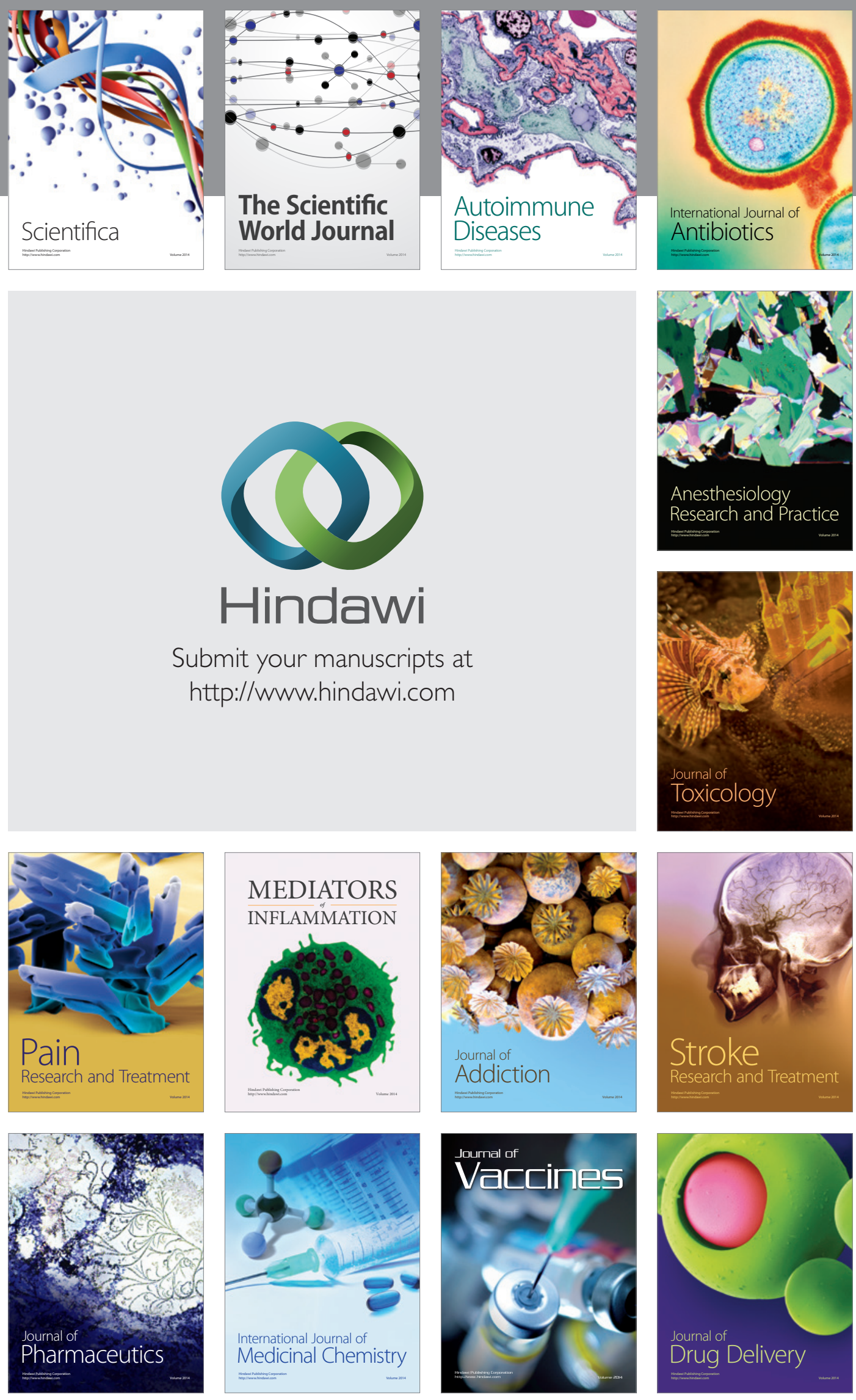